Please do not remove this page

RMIT

UNIVERSITY

\title{
Comparison of photon counting and analog techniques for the measurement of photon pair generation in a PPLN waveguide
}

Castelletto, Stefania; Degiovanni, Ivo; Schettini, Valentina; Del Rosso, Tommaso; Margheri, Giancarlo; Papi, Leonardo; Sottini, Stefano

https://researchrepository.rmit.edu.au/esploro/outputs/9921858804201341/filesAndLinks?institution=61RMIT_INST\&index=null

Castelletto, S., Degiovanni, I., Schettini, V., Del Rosso, T., Margheri, G., Papi, L., \& Sottini, S. (2008).

Comparison of photon counting and analog techniques for the measurement of photon pair generation in a PPLN waveguide. Journal of the Optical Society of America B-Optical Physics, 25(1), 7-14.

https://doi.org/10.1364/josab.25.000007

Document Version: Published Version

Published Version: https://doi.org/10.1364/josab.25.000007

Repository homepage: https://researchrepository.rmit.edu.au

(C) 2007 Optical Society of America

Downloaded On 2023/04/26 23:04:32 +1000 
Thank you for downloading this document from the RMIT Research Repository.

The RMIT Research Repository is an open access database showcasing the research outputs of RMIT University researchers.

RMIT Research Repository: http://researchbank.rmit.edu.au/

\section{Citation:}

Castelletto, S, Degiovanni, I, Schettini, V, Del Rosso, T, Margheri, G, Papi, L and Sottini, S 2008, 'Comparison of photon counting and analog techniques for the measurement of photon pair generation in a PPLN waveguide', Journal of the Optical Society of America B-Optical Physics, vol. 25, no. 1, pp. 7-14.

See this record in the RMIT Research Repository at:

http://researchbank.rmit.edu.au/view/rmit:21952

Version: Published Version

Copyright Statement: (c) 2007 Optical Society of America

Link to Published Version:

http://dx.doi.org/10.1364/josab.25.000007 


\title{
Comparison of photon counting and analog techniques for the measurement of photon pair generation in a PPLN waveguide
}

\author{
Stefania Castelletto, ${ }^{1}$ Ivo Pietro Degiovanni, ${ }^{2, *}$ Valentina Schettini, ${ }^{2}$ Tommaso Del Rosso, ${ }^{3}$ Giancarlo Margheri, ${ }^{3}$ \\ Leonardo Papi, ${ }^{3}$ and Stefano Sottini ${ }^{3,4}$ \\ ${ }^{1}$ Physics School, Melbourne University, Victoria, Australia \\ ${ }^{2}$ Istituto Nazionale di Ricerca Metrologica, Strada delle Cacce 91-10135 Torino, Italy \\ ${ }^{3}$ Istituto dei Sistemi Complessi CNR, Sesto Fiorentino, Italy \\ ${ }^{4}$ E-mail: stefano.sottini@isc.cnr.it \\ *Corresponding author: degio@inrim.it
}

Received June 14, 2007; accepted October 11, 2007;

posted November 6, 2007 (Doc. ID 84147); published December 11, 2007

\begin{abstract}
We present two independent measurements of the photon pairs production efficiency (PPPE) at $1572 \mathrm{~nm}$, generated in a noncommercial periodically poled lithium-niobate waveguide fabricated in our laboratory. The first measurement, referred to as "direct" measurement, is performed at the photon-counting level (light power at the level of a few picowatts), exploiting a typical coincidence detection technique and a dedicated statistical model. In this case the measured PPPE is $(4.1 \pm 1.1) 10^{11}$ pairs/(s W). The same parameter was estimated independently by a well-established "indirect" measurement, based on a difference frequency generation experiment (typical light power level of a few microwatts). This other measurement yields $(5.0 \pm 2.4) 10^{11}$ pairs $/(\mathrm{s} \mathrm{W})$. Despite the large uncertainty of this second measurement, we observe that the two results are in good agreement even considering only the lower uncertainty value. To our knowledge, it is the first realization of a comparison between these two measurement techniques, working at so different light levels. () 2007 Optical Society of America
\end{abstract}

OCIS codes: $190.4410,270.5290,130.2790,130.4310,030.5260$.

\section{INTRODUCTION}

Parametric downconversion (PDC) produces light with a two-photon field description [1] from the interaction of a pump laser in a nonlinear crystal. This two-photon light, which allows one photon to indicate or herald the existence of its twin, gained popularity in applications such as quantum radiometry [2] and quantum information [3,4]. In fact it has been proved as a tool for direct photoncounting detector calibration without any external standard and a more suitable single photon source than an attenuated laser, though maintaining low cost and almost on-the shelf access [5,6]. However, in spite of present PDC practicality, before an actual application to metrology and to quantum information will be established, further improvements of the source are required, e.g., broader wavelength coverage, single mode generation, high efficiency of photon pair production. This is mostly true on the side of certain quantum information processing applications [4], where a key demand is an efficient production of photon pairs for the low pump power level injected.

For high efficiency of pair production, and single photons produced at the telecom wavelength, the use of periodically poled crystals is becoming common [7-10].

The advantage of using periodically poled crystals is based on the increment of the correlated photons generated, so that the photon pairs do not suffer from excessive post-selection filtering, and rely on the absence of walkoff, which is another limitation encountered in bulk crystals. In fact, in a periodically poled material, an higher
PDC pair production efficiency is guaranteed by noncritical quasi-phase matching (QPM), obtainable in each period of the material at a given temperature, making it possible to utilize the largest value of the effective nonlinear coefficient.

The modal PDC emission in free propagation is still not optimal, because to have the heralded photons in welldefined spectral and spatial modes, spatial and temporal modal post selection of PDC photons is adopted, e.g., single-mode fiber coupling together with narrowband spectral filtering. Post selection limits the advantage of having produced a higher rate of photon pairs and increases the complexity in the optimization of mode matching among the pump and the PDC photons modes [11-14].

Waveguides (WGs) have been used instead of the post selection, maintaining the high level of photon creation [15-22], and waveguided PDC is one route to decorrelate the spatial degree of freedoms from spectral ones and to eliminate at the same time the spatial correlations between the PDC photons. In this case correlated photons are generated in single modes directly and open up the possibility of having multiplexed sources of PDC in a relatively small shape.

The most convenient configuration for having a single photon source at telecom wavelength is with one heralding photon of the pair in the visible, where single photon detectors are more efficient [19]. However PDC photon pairs at $1550 \mathrm{~nm}$ can be interesting not only because periodically poled lithium-niobate waveguides (PPLN WGs) 
are more easily produced with a longer period, but also because, as recently pointed out by Chen et al. [23], the role of photon sources able to generate identical photons in well-defined spatiotemporal modes is greatly important for linear optical quantum computing applications [24]. Efforts in this direction have been pursued lately [20] by using a pulsed pump where, however, the multiple photon pair generation has to be carefully controlled.

In this paper we tested a noncommercial PPLN WG fabricated in our laboratory to determine its photon pair production efficiency (PPPE) in a PDC process. The aim is to validate our measurement technique based on coincidence detection (CD) and statistical reconstruction (which we will refer to as "direct" measurement technique) by comparing its evaluation of PPPE with the one obtained with a well-established ("indirect") method based on difference frequency generation (DFG) [25]. The direct photon-counting technique, based on $\mathrm{CD}$, presents a unique feature with respect to the measurements reported by Baldi et al. [25], which are based on the direct measuring of the PDC emission intensity. This is due to the fact that the CD technique allows the discrimination of the photon pairs produced by the PDC process from the single photons that are a result of other fluorescence effects. For example, only with CD measurement would it could it be possible to get the correct parametric gain even in the case $\mathrm{Er}^{3+}$ dopant, which is widely used in fibers and $\mathrm{LiNbO}_{3}$ self-doubling systems [26] and is highly fluorescent at the telecom wavelengths.

The two measurement techniques that we used exploit similar experimental apparatus. In the indirect technique based on DFG, the amplification of the seed field was measured by calibrated analog infrared detectors. The typical light power measured in this case was of the order of a few microwatts. The setup for the direct measurement technique is straightforward from the DFG setup, simply by suppressing the input seed and replacing analog detectors with the photon-counting apparatus, to detect directly coincidence counts from photon pairs. The light power level in this case was of the order of a few picowatts. Despite the wide analogy between the two experimental schemes, to our knowledge, it is the first realization of a comparison between these two measurement techniques, working at so different light power levels. From the experimental point of view, the DFG measurement can be helpful in setting up the PDC apparatus.

Section 2 is devoted to the presentation of the experimental setup used to perform the direct measurement of PPPE from PDC produced in a PPLN WG, and we analyze the results according to our statistical model. In Section 3 the indirect measurement technique based on a DFG experiment is presented, and the results are discussed.

\section{DIRECT MEASUREMENT TECHNIQUE BASED ON COINCIDENCE DETECTION OF PDC PHOTON PAIRS}

Our PDC source is based on an annealed proton exchanged (APE), $17 \mathrm{~mm}$ long WG realized on a PPLN crystal. The ferroelectric domains of the crystal were inverted using the standard electric poling technique [27], and several channel WGs with width ranging from $2 \mu \mathrm{m}$ up to $12 \mu \mathrm{m}$ were subsequently fabricated on the PPLN using the same APE technique, which is outlined, in the planar case, in several papers $[28,29]$ apart from the added lithographic step. The PPLN-APE channel WG used has a total width of $8.4 \mu \mathrm{m}$, a depth of $3.62 \mu \mathrm{m}$, and a poling period of $16.3 \mu \mathrm{m}$, and it realizes a degenerate PDC peaked at $1572 \mathrm{~nm}$ at a temperature close to $50^{\circ} \mathrm{C}$.

Figure 1 shows the experimental setup used to perform the PPPE measurement based on CD of PDC photon pairs. The pump radiation, supplied by a cw diode laser at $786 \mathrm{~nm}$, is focused onto the WG by a microscope objective (Obj) $20 \times$. The temperature of the WG is kept constant within $0.1^{\circ} \mathrm{C}$ by an active feedback circuit connected to a Peltier cell. At the output of the PPLN device PDC photons are collected and collimated by another $20 \times$ microscope objective and eventually coupled to a single-mode fiber by an aspheric lens (L), $8 \mathrm{~mm}$ focal length and $0.5 \mathrm{NA}$, after filtering out the pump laser by a dichroic mirror (DM2) and long-pass filters (HBPs). The pump reflected by the dichroic mirror is directed toward a calibrated silicon detector. During the alignment process a CCD camera was used to monitor the excitation of the WG fundamental mode at the pump wavelength. Also, a seed field at

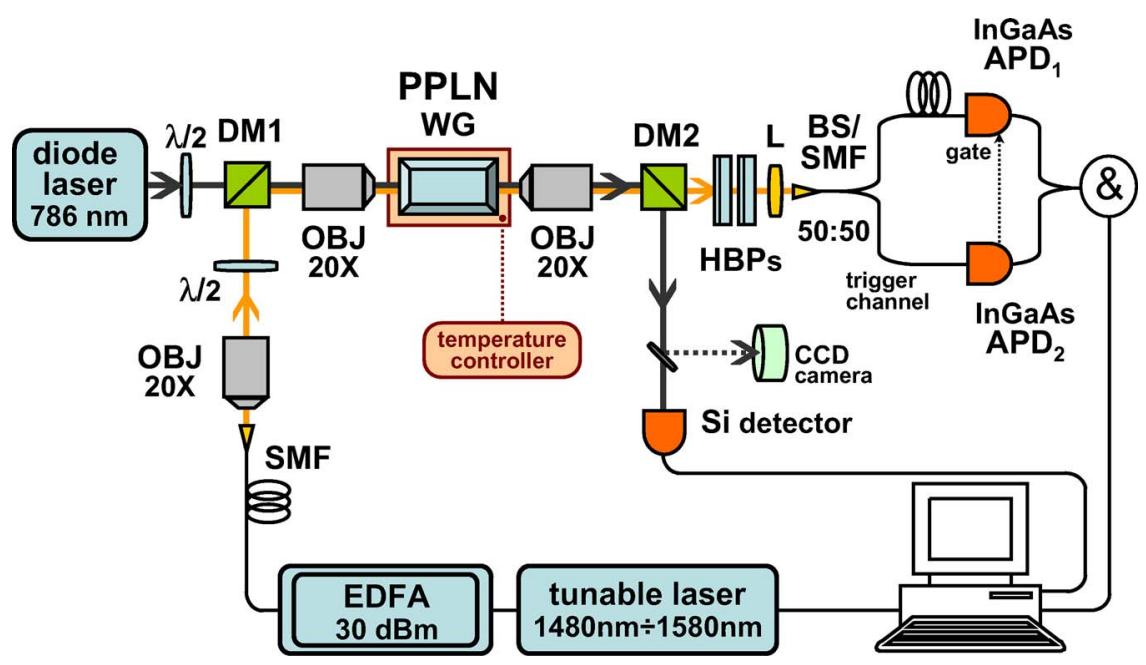

Fig. 1. (Color online) Setup used to generate photon pairs at $1572 \mathrm{~nm}$ from a cw pumped parametric down-conversion in a WG. 
$1572 \mathrm{~nm}$ wavelength is coupled in the guide to simplify the alignment and the maximization of the collection of the PDC photons into the single-mode fiber (SMF). The PDC photons are routed to an all-fiber 50:50 beam splitter (BS/SMF), whose outputs are sent to single photon counters, SPDM Id 200 by Id Quantique based on InGaAs avalanche photodiodes $\left(\mathrm{APD}_{1,2}\right)$ operating in gated mode and with an internal active quenching circuit. Because of high dark counts and high afterpulsing effects, detector $\mathrm{APD}_{1}$ (triggered detector) is gated by the $\mathrm{APD}_{2}$ (trigger detector), and thus maintains its gating frequency sufficiently low (a few kilohertz). $\mathrm{APD}_{2}$ is gated by its internal gate (typically $100 \mathrm{KHz}$ ), with a measurement time window $T$ of 100 ns. Because of the afterpulsing, both detectors were set with the highest possible deadtime of $10 \mu \mathrm{s}$.

A time-to-amplitude converter and a multichannel analyzer were used to visualize the coincidences and to discriminate the true coincidences from the accidental ones (see Fig. 2). The overall coincidence window $w$ is given by the $\mathrm{APD}_{1}$ measurement time window of $20 \mathrm{~ns}$.

According to our statistical model whose detailed presentation is shown in Appendix A, the mean rate of photon pairs production is

$$
\Lambda_{c}=\frac{p_{c}^{\text {true }}}{2 \xi_{B S}\left(1-\xi_{B S}\right) \pi_{w, \mathrm{I}} \eta_{1} \eta_{2} \tau_{1} \tau_{2} T}
$$

where $T$ is the gate time width and $\tau_{2}$ is the overall optical transmittance of the photons in the trigger channel, while $\tau_{1}$ is the transmittance of the triggered channel. $\eta_{i}$ $(i=1,2)$ indicates the detection efficiency of the $i$ th detector $\left(\mathrm{APD}_{i}\right) . \xi_{B S}$ is the splitting factor of the BS/SMF, the term $2 \xi_{B S}\left(1-\xi_{B S}\right)$ accounts for the probability that the two photons of a pair are split one for each output arm of the BS/SMF. $p_{c}^{\text {true }}$ is the probability of true coincidences per gate. We underline that our statistical model accounts for the presence of more than one pair in the gate time interval T. According to Fig. $2, p_{c}^{\text {true }}$ is evaluated as the ratio between the measured true coincidence counts $N_{c}^{\text {true }}$ (the coincidence counts belonging to the "peak") and the

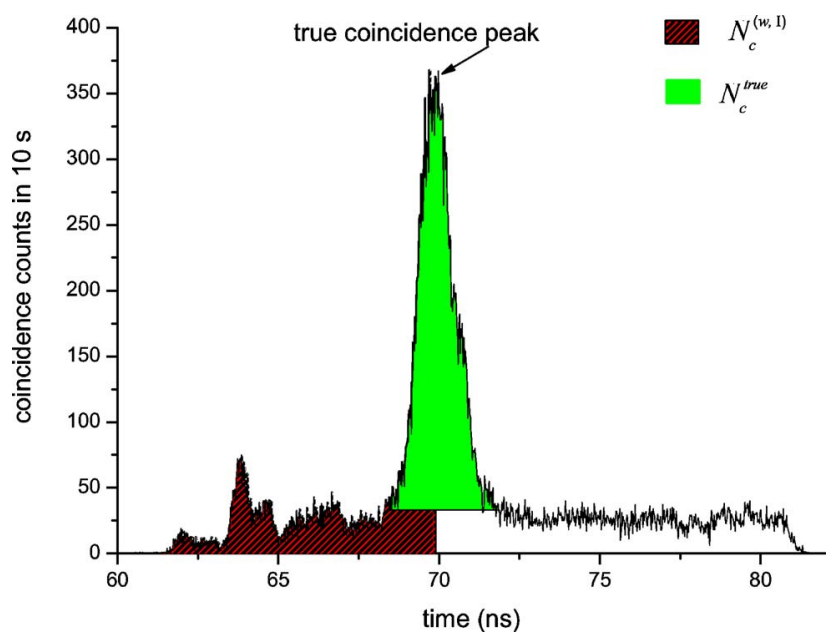

Fig. 2. (Color online) Coincidence counts histogram in the experimental condition of $T=20 \mathrm{~ns}$, with triggering gate rate $100 \mathrm{KHz}$. Coincidence fluctuations before the peak are due to electronic imperfections. We account for these fluctuations in the estimation of $\pi_{w, \mathrm{I}}$. number of gating events $N_{\text {gate }}$ of the trigger channel, i.e., $p_{c}^{\text {true }}=N_{c}^{\text {true }} / N_{\text {gate }} . \pi_{w, \mathrm{I}}$ is the probability of the triggered detector not firing for an accidental coincidence in the first half of the coincidence window, before the arrival of the correlated photon of the pair. According to Fig. 2, it can be estimated as $\pi_{w, \mathrm{I}}=1-N_{c}^{(w, \mathrm{I})} / N_{\text {gate }}$ where $N_{c}^{(w, \mathrm{I})}$ are the accidental coincidence counts in the first half of the coincidence window.

We measured the coincidence counts for several pump power levels, effectively coupled to the WG and responsible for the pairs production, to estimate the PPPE.

In order to estimate the optical losses in our experimental setup, we used the tunable diode laser aligned in the WG channel and measured the optical transmittance for each component along the optical path (i.e., the waveguide and its output face, the microscope objective, the dichroic mirror, filters, lens, and fibers) as well as SMF matching with the WG modes. On the trigger arm we measured a total transmittance $\tau_{2}=(0.197 \pm 0.026)$, while on the triggered arm $\tau_{1}=(0.181 \pm 0.022)$. We also checked the splitting factor $\xi_{B S}$ of the fiber integrated 50:50 BS/ SMF, obtaining $\xi_{B S}=(0.50 \pm 0.01)$. The measurements were repeated several times during the experiment, and the final uncertainty value accounts for the uncertainty in the optimization of the alignment, for the data statistical fluctuations, as well as for the uncertainty in the calibration of the analog detectors used.

To evaluate the actual quantum efficiency of the two single photon detectors SPDM Id 200 by Id Quantique, we have to consider that our PDC source presents large bandwidth. We did not use any interference filter, and the fiber lens collection aperture was matching the WG mode so that all the spectral components were collected. For this reason we estimated an effective mean quantum efficiency for the detection of the pairs accounting for the wavelength dependence of the quantum efficiency according to

$$
\eta_{1} \eta_{2}=\int f\left(\lambda_{1}\right) \eta_{1}\left(\lambda_{1}\right) \eta_{2}\left(\lambda_{2}\right) \delta\left(\frac{1}{\lambda_{1}}+\frac{1}{\lambda_{2}}-\frac{1}{\lambda_{p}}\right) \mathrm{d} \lambda_{1} \mathrm{~d} \lambda_{2},
$$

where $\eta_{i}\left(\lambda_{i}\right)$ is the quantum efficiency of each detector at the wavelength $\lambda_{i} \cdot \eta_{i}\left(\lambda_{i}\right)$ is obtained from the results of our detectors calibration [30], as well as from the typical detection efficiency spectral curve reported by the detectors datasheet [31]. The delta function accounts for the energy conservation of the two photons of the pair; finally, $f\left(\lambda_{1}\right)$ is the spectral distribution of the PDC light, assumed to be Gaussian with FWHM $\Delta \omega_{\text {FWHM }}$.

$\Delta \omega_{\mathrm{FWHM}}$ is calculated in the degenerate case as [25]

$$
\Delta \omega_{\mathrm{FWHM}}=\sqrt{\frac{2 \pi c}{4 \frac{\partial n_{\text {eff }}}{\partial \omega}+2 \omega_{Q P M} \frac{\partial^{2} n_{\text {eff }}}{\partial \omega^{2}} \mid},},
$$

where $L$ is the length of the WG, $\omega_{Q P M}$ is the frequency of photon at phase matching, and $n_{\text {eff }}(\omega)$ is the effective index of the WG at frequency $\omega$. Utilizing the well known effective-index method [32], we theoretically estimate a value of $\Delta \omega_{\mathrm{FWHM}}$ of $(45 \pm 7) \mathrm{THz}$. In Fig. 3 is shown a comparison between the calculated (according to the effectiveindex method) and the measured near-field intensity dis- 

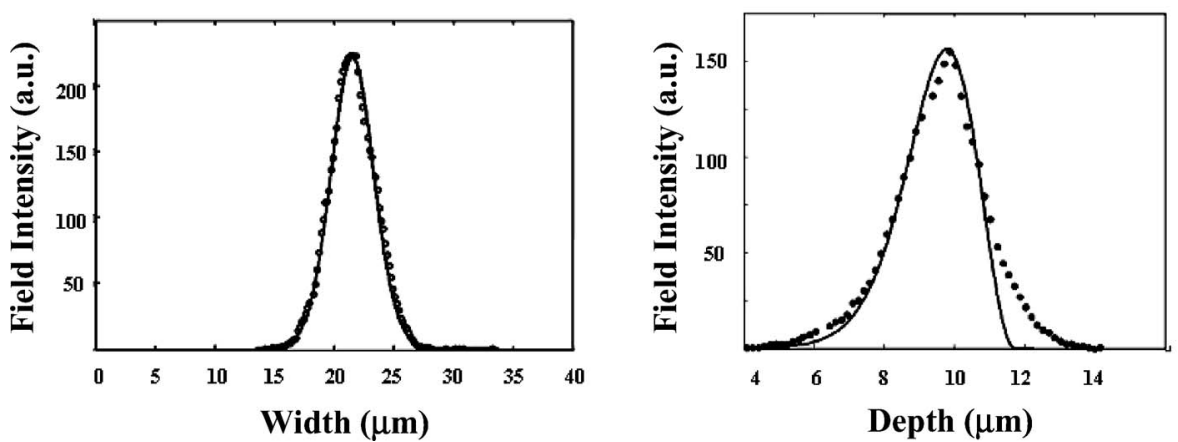

Fig. 3. Calculated (solid curve) and measured (dots) near field intensity distributions at the WG output.

tributions exiting the WG. The optimum agreement between the theory and the experimental data is an indirect confirmation of the validity of the model used.

Thus, our analysis leads to $\eta_{1} \eta_{2}=(0.0047 \pm 0.0005)$, where we accounted for the uncertainty in the calibration of our detectors discussed above, as well as contributions coming from our theoretical estimation of the PDC light bandwidth.

According to Eq. (1), the measured photon pairs rates $\Lambda_{c}$ are $(1.35 \pm 0.32) 10^{7} \mathrm{pairs} / \mathrm{s},(2.76 \pm 0.65) 10^{7} \mathrm{pairs} / \mathrm{s}$, and $(9.9 \pm 2.4) 10^{7}$ pairs/s, for a guided pump power of, respectively, $(33 \pm 4) \mu \mathrm{W},(69 \pm 7) \mu \mathrm{W}$, and $(230 \pm 23) \mu \mathrm{W}$. The guided pump power, $P_{p}(0)$, was measured by the silicon detector. According to Fig. 1 we calibrated the total path transmittance toward the silicon detector obtaining $\tau_{\mathrm{Si}}=(0.122 \pm 0.012)$.
A reasonable parameter to estimate the performance of a PPLN WG is the PPPE calculated as the ratio between the measured photon pairs rate and the corresponding guided pump power,

$$
\operatorname{PPPE}=\frac{\Lambda_{c}}{P_{p}(0)} .
$$

We performed a detailed uncertainty analysis for each measurement according to metrological guidelines [33], consisting essentially in Gaussian propagation of uncertainty. As an example, in Table 1 we present the uncertainty analysis associated with the PPPE measurement with guided pump power $P_{p}(0)=33 \mu \mathrm{W}$. We underline that the main contributions of uncertainty come from the difficulties in the evaluation of the total transmittance of

Table 1. Uncertainty Contributions in the Direct Measurement of PPPE

\begin{tabular}{|c|c|c|c|c|c|}
\hline Quantity & Value & $\begin{array}{l}\text { Standard } \\
\text { Uncertainty }\end{array}$ & $\begin{array}{l}\text { Deg. of } \\
\text { Freedom }\end{array}$ & $\begin{array}{l}\text { Sensitivity } \\
\text { Coefficient }\end{array}$ & $\begin{array}{c}\text { Uncertainty } \\
\text { Contribution } \\
\left.\quad / \frac{\text { pairs }}{\mathrm{s} \mathrm{W}}\right]\end{array}$ \\
\hline$N_{c}^{\text {true }}$ & 1104 & 27 & 5 & $3.7410^{8} \frac{\text { pairs }}{\mathrm{s} \mathrm{W}}$ & $1.0110^{10}$ \\
\hline$N_{\text {gate }}$ & 9742141 & 927 & 5 & $-4.2410^{4} \frac{\text { pairs }}{\mathrm{s} \mathrm{W}}$ & $-3.9310^{7}$ \\
\hline$N_{c}^{(w, \mathrm{I})}$ & 384 & 12 & 5 & $4.2410^{4} \frac{\text { pairs }}{\mathrm{s} \mathrm{W}}$ & $4.9010^{5}$ \\
\hline$\tau_{1}$ & 0.181 & 0.022 & $\infty$ & $-2.2810^{12} \frac{\text { pairs }}{\mathrm{s} \mathrm{W}}$ & $-4.9310^{10}$ \\
\hline$\tau_{2}$ & 0.197 & 0.026 & $\infty$ & $-2.0910^{12} \frac{\text { pairs }}{\mathrm{s} \mathrm{W}}$ & $-5.3510^{10}$ \\
\hline$\xi_{B S}$ & 0.50 & 0.01 & $\infty$ & $0^{(*)} \frac{\text { pairs }}{\mathrm{s} \mathrm{W}}$ & $0^{(*)}$ \\
\hline$\eta_{1} \eta_{2}$ & 0.0047 & 0.0005 & $\infty$ & $-8.7910^{13} \frac{\text { pairs }}{\mathrm{s} \mathrm{W}}$ & $-4.3910^{10}$ \\
\hline$T$ & $10^{-7} \mathrm{~s}$ & $10^{-9} \mathrm{~s}$ & $\infty$ & $-4.1310^{18} \frac{\text { pairs }}{\mathrm{s}^{2} \mathrm{~W}}$ & $-4.1310^{3}$ \\
\hline$P_{\mathrm{Si}}(0)$ & $3.9810^{-6} \mathrm{~W}$ & $0.0410^{-6} \mathrm{~W}^{33}$ & 5 & $-1.0410^{17} \frac{\text { pairs }}{\mathrm{s} \mathrm{W}^{2}}$ & $-4.1510^{9}$ \\
\hline$\tau_{\mathrm{Si}}$ & 0.122 & 0.012 & $\infty$ & $3.3910^{12} \frac{\text { pairs }}{\mathrm{s} \mathrm{W}}$ & $4.0610^{10}$ \\
\hline PPPE & $4.1310^{11} \frac{\text { pairs }}{\mathrm{s} W}$ & & $>100$ & & $1.0810^{11}$ \\
\hline
\end{tabular}

${ }^{a}$ The Sensitivity Coefficient shows the relationship between the individual standard uncertainty component to the standard uncertainty and the reported value of PPPE, according to the well known gaussian propagation of uncertainty formula. The degrees of freedom represent the statistical degrees of freedom related to the estimation of standard uncertainty component [33]. ${ }^{(*)}$ The uncertainty contribution associated to $\xi_{B S}$ is $2.3610^{10}$ pairs s$~^{-1} \mathrm{~W}^{-1}$ is obtained accounting for the higher order terms in the uncertainty expansion according to the ISO guideline [33] 


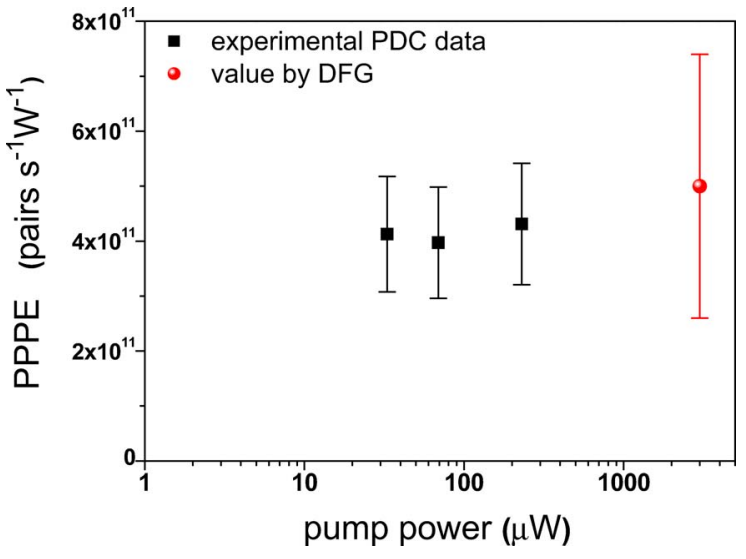

Fig. 4. (Color online) Photon pairs production efficiency versus coupled pump power for the PDC experiment (squares) and the DFG experiment (dot).

each channel because of the reproducibility of the fiber coupling and the presence of many optical components.

By averaging the three measurements, plotted in Fig. 4 , we thus obtained a $\operatorname{PPPE}=(4.1 \pm 1.1) 10^{11}$ pairs $/(\mathrm{s} \mathrm{W})$. This PPPE value is compared with the predicted value obtained by the DFG experiment described in Section 3.

\section{INDIRECT MEASUREMENT TECHNIQUE BASED ON DIFFERENCE FREQUENCY GENERATION}

According to Baldi et al. [25], we evaluated the PPPE by performing an experiment of DFG, whose experimental setup is reported in Fig. 5.

The setup is similar to the one in Fig. 1, with the pump at $786 \mathrm{~nm}$, except that in this case the tunable laser at $1572 \mathrm{~nm}$ was used as a seed to generate the difference frequency idler power $\left(P_{i}\right)$. The guided pump power is monitored by a silicon calibrated detector at the exit of the WG as in the previous experiment. The seed interacting in the WG is also amplified; $\Delta P_{s}=P_{s}(L)-e^{-\alpha_{s} L} P_{s}(0)$ is the amplified signal power. We measured the sum of the generated idler power and the amplified signal power $\Delta P_{s}+P_{i}$ by a lock-in amplifier and a calibrated germanium detector. According to Baldi et al. [25], near degeneracy $\Delta P_{s}+P_{i}$ is given by

$$
\Delta P_{s}+P_{i} \cong 2 P_{s}(L) g^{2} L^{2}\left(1-\frac{\alpha_{p} L}{2}\right),
$$

where $P_{s}(L)\left(P_{s}(0)\right)$ is the signal power at the output (input) of the WG of length $L, \alpha_{p}\left(\alpha_{s}\right)$ is the loss coefficient at the pump (signal) wavelength, and $g$ is the parametric gain. Relation (5) holds only in the case of low losses inside the WG both at the signal and at the pump wavelengths. To ensure its validity, we performed the measurement of the WG losses using the Fabry-Perot method [34]. By the field intensity profiles of Fig. 3, we evaluated $\alpha_{p}=(0.57 \pm 0.04) \mathrm{cm}^{-1}$ and $\alpha_{s}=(0.18 \pm 0.03) \mathrm{cm}^{-1}$.

In Fig. 6 we plot the relative measurements of $\Delta P_{s}$ $+P_{i}$ versus the temperature of the $\mathrm{WG}$ at the fixed signal wavelength of $1572 \mathrm{~nm}$. We observe that the signal and idler production is maximum at $53^{\circ} \mathrm{C}$.

By absolute measurements of $\Delta P_{s}+P_{i}$ versus the pump power (Fig. 7) at the entrance of the WG, for fixed $P_{s}(0)$, we deduced the parametric gain value $g / \sqrt{P_{p}(0)}$ $=(24 \pm 4) \mathrm{m}^{-1} \mathrm{~W}^{-1 / 2}$. This value is comparable with a previously obtained value in a similar WG (see Table III in the paper by Baldi et al. [25]).

The signal power produced by spontaneous PDC is predicted to be [25]

$$
P_{s}^{\mathrm{PDC}}=\frac{\hbar \omega_{s}}{2 \pi} e^{-\alpha_{s} L} g^{2} L^{2}\left[\frac{1-e^{-\alpha_{p} L / 2}}{\alpha_{p} L / 2}\right]^{2} \Delta \omega_{\mathrm{FWHM}},
$$

where $g$ is the same gain parameter of the DFG experiment and $\Delta \omega_{\mathrm{FWHM}}$ is the signal bandwidth at FWHM calculated according to Eq. (3).

Therefore, the PPPE $=\Lambda_{c} / P_{p}(0)$ is

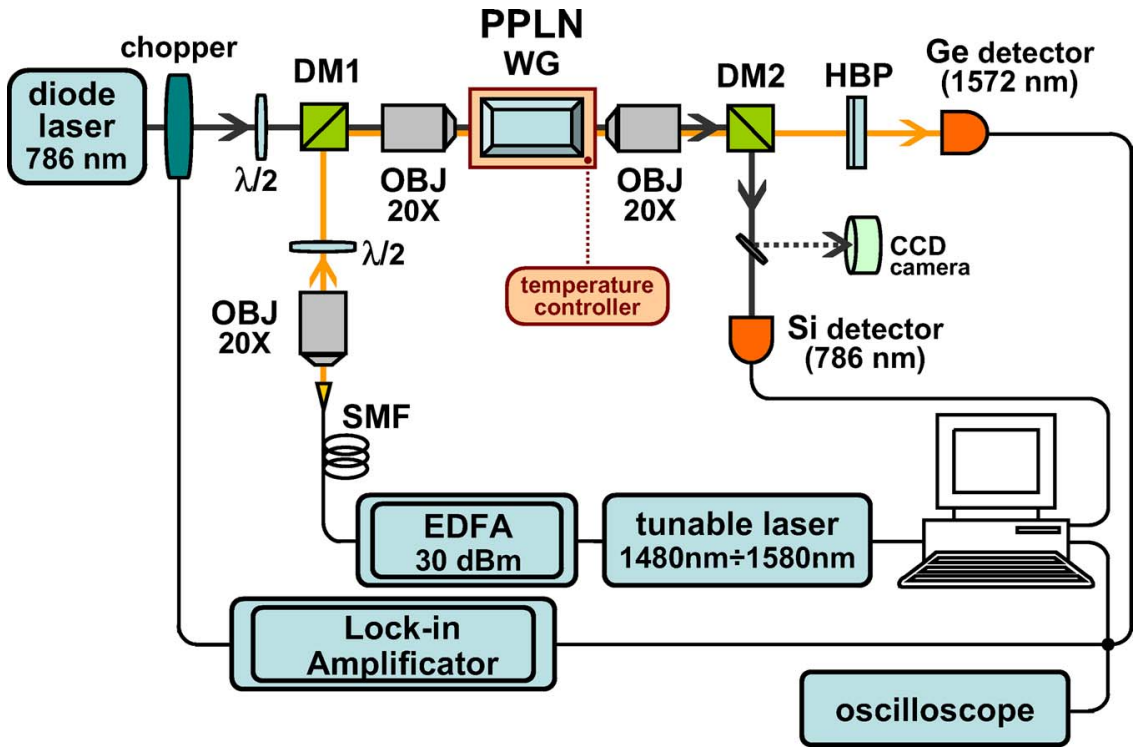

Fig. 5. (Color online) Setup used for realizing DFG at $1572 \mathrm{~nm}$ in a WG. 


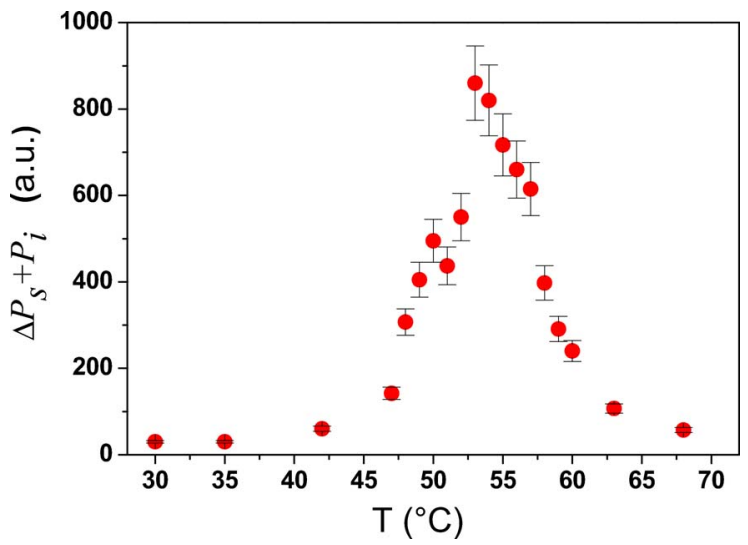

Fig. 6. (Color online) Measured value in arbitrary units of $\Delta P_{s}$ $+P_{i}$ at $1572 \mathrm{~nm}$ versus the temperature of the WG.

$$
\mathrm{PPPE}=\frac{1}{2 \pi \tau_{W G}} \frac{g^{2}}{P_{p}(0)} L_{e f f}^{2}\left[\frac{1-e^{-\alpha_{p} L / 2}}{\alpha_{p} L / 2}\right]^{2} \Delta \omega_{\mathrm{FWHM}},
$$

where $L_{\text {eff }}=(13 \pm 2) \mathrm{mm}$ is the measured effective length of PPLN APE WG [35], whereas the total length is $L$ $=(17.0 \pm 0.1) \mathrm{mm}$. The transmittance of the output polished face of the WG is estimated from the well known properties of the interface $\mathrm{LiNbO}_{3}$-air as $\tau_{W G}$ $=(0.8685 \pm 0.0100)$. The estimated PPPE in this case is $(5.02 \pm 2.41) 10^{11}$ pairs/(s W). The analysis of the uncertainty contributions in this case is presented in Table 2. In this case the main contributions of uncertainty come from the evaluation of $L_{e f f}, g / \sqrt{P_{p}(0)}$, and $\Delta \omega_{\mathrm{FWHM}}$ because of the very indirect techniques used for their estimations [35].

\section{CONCLUSIONS}

In this paper we measured the PPPE from PDC generated in a noncommercial PPLN WG fabricated in our laboratory. The tests were performed at $1572 \mathrm{~nm}$ by two different methods. The first one is based on CD technique at the photon-counting level, which allows us, by means of a dedicated statistical analysis and loss estimation, to reconstruct directly the rate of pairs production. The second one [25] is an indirect method, that allows the estimation of the PPPE from the results of a DFG experiment.

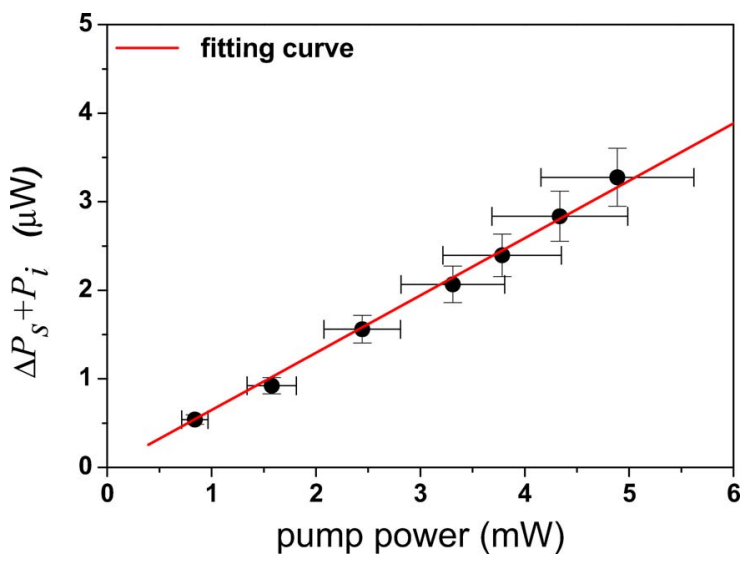

Fig. 7. (Color online) Measured value of $\Delta P_{s}+P_{i}$ at $1572 \mathrm{~nm}$ versus input pump power.
Thanks to the theoretical results reported in Baldi et al. [25], the DFG experiment was utilized to validate our photon-counting technique, including a dedicated statistical analysis.

In the experimental setup of the CD technique, the probability of detecting a true coincidence count for each $\mathrm{PDC}$ pair produced is around $8 \times 10^{-5}$. We underline that, despite this low probability of coincidence detection per PDC photon pair (due mainly to the poor quantum efficiency of the single photon detectors), in this paper we proved that our coincidence-detection measurement technique offers an absolute, reliable, and direct measurement of the produced photon rate as well as of the parametric gain.

Although our direct technique is, at first glance, similar to direct measurement of PDC light reported in Baldi et al. [25], we emphasize that our direct photon-counting technique based on CD presents a unique feature. As already noticed, only the $\mathrm{CD}$ technique is able to get the correct parametric gain even in the case of $\mathrm{Er}_{3+}$ dopant, widely used in fibers and $\mathrm{LiNbO}_{3}$ self doubling systems [26], which is highly fluorescent at the telecom wavelengths.

In conclusion, to our knowledge, this is the first realization of a comparison between direct photon-counting and DFG techniques, together with a rigorous analysis of the contributions leading to the final uncertainty associated with PPPE values. According to Fig. 4, the PPPE values obtained with the two measurements are in good agreement, even if we consider only the lower uncertainty associated with the PDC experiment. This guarantees the reliability of the absolute and direct technique and of the associated statistical model. The point of view is strongly metrological in order to provide a standard method for evaluating the parametric gain of guiding optical devices.

\section{APPENDIX A: STATISTICAL MODEL FOR THE COINCIDENCE DETECTION TECHNIQUE}

This appendix presents the statistical model that we developed to describe the measurements results of the coincidence detection setup drawn in Fig. 1. The main result of this statistical model is Eq. (1), which is used to obtain the value of PPPE from the direct measurement technique.

The trigger detector may click for the arrival of a photon of the pair, which can trigger a true coincidence $\left(p_{2}\right)$, or it may click for an event that can trigger only accidental coincidence $\left(p_{2}^{a c c}\right)$ because, e.g., it comes from a dark count, or from stray light, or from photon pairs whose correlated photon has taken the same path after the BS, or it has been lost because of optical losses. Thus, for each internal gate pulse, we can write the total probability of $\mathrm{APD}_{2}$ to click as

$$
p_{2}^{t o t}=p_{2}+p_{2}^{a c c} .
$$

We focus our attention on the term $p_{2}$, 
Table 2. Uncertainty Contributions in the Indirect Measurement of PPPE

\begin{tabular}{|c|c|c|c|c|c|}
\hline Quantity & Value & $\begin{array}{c}\text { Standard } \\
\text { Uncertainty }\end{array}$ & $\begin{array}{l}\text { Deg. of } \\
\text { Freedom }\end{array}$ & $\begin{array}{l}\text { Sensitivity } \\
\text { Coefficient }\end{array}$ & $\begin{array}{c}\text { Uncertainty } \\
\text { Contribution } \\
\left.\quad / \frac{\text { pairs }}{\mathrm{s} \mathrm{W}}\right]\end{array}$ \\
\hline$\tau_{\mathrm{WG}}$ & 0.8685 & 0.0100 & $\infty$ & $-5.810^{11} \frac{\text { pairs }}{\mathrm{s} \mathrm{W}}$ & $-5.8110^{9}$ \\
\hline$L_{e f f}$ & $1.310^{-2} \mathrm{~m}$ & $0.210^{-2} \mathrm{~m}$ & $\infty$ & $7.710^{13} \frac{\text { pairs }}{\mathrm{s} \mathrm{W} \mathrm{m}}$ & $1.5510^{11}$ \\
\hline$L$ & $1.710^{-2} \mathrm{~m}$ & $0.0110^{-2} \mathrm{~m}$ & $\infty$ & $-1.310^{13} \frac{\text { pairs }}{\mathrm{s} \mathrm{W} \mathrm{m}}$ & $-1.3210^{9}$ \\
\hline$g / \sqrt{P_{p}(0)}$ & $24 \mathrm{~m}^{-1} \mathrm{~W}^{-1 / 2}$ & $4 \mathrm{~m}^{-1} \mathrm{~W}^{-1 / 2}$ & $\infty$ & $410^{10} \frac{\text { pairs } \mathrm{m}}{\mathrm{s} \mathrm{W}^{1 / 2}}$ & $1.6810^{11}$ \\
\hline$\alpha_{p}$ & $57 \mathrm{~m}^{-1}$ & $4 \mathrm{~m}^{-1}$ & $\infty$ & $3.910^{9} \frac{\text { pairs m }}{\mathrm{s} \mathrm{W}}$ & $-1.5810^{10}$ \\
\hline$\Delta \omega_{\mathrm{FWHM}}$ & $4.510^{13} \mathrm{~Hz}$ & $710^{12} \mathrm{~Hz}$ & $\infty$ & $0.01 \frac{\text { pairs }}{W}$ & $7.8410^{10}$ \\
\hline PPPE & $5.0210^{11} \frac{\text { pairs }}{\mathrm{sW}}$ & & $\infty$ & & $2.4110^{11}$ \\
\hline
\end{tabular}

$$
p_{2}=\sum_{n=0}^{\infty} \sum_{k=0}^{n}\left[1-\left(1-\eta_{2}\right)^{k}\right] \mathcal{B}\left(k \mid n ; 2 \xi_{B S}\left(1-\xi_{B S}\right) \tau_{1} \tau_{2}\right) \mathcal{P}\left(n \mid \Lambda_{c} T\right)
$$

Here $\mathcal{P}\left(n \mid \Lambda_{c} T\right)$ is the probability of having $n$ photon pairs, with mean rate $\Lambda_{c}$ during the time interval $T$. $\mathcal{B}\left(k \mid n ; 2 \xi_{B S}\left(1-\xi_{B S}\right) \tau_{1} \tau_{2}\right)$ is a binomial distribution accounting for the probability that, given $n$ pairs produced, only in $k$ cases both photons of the pair are transmitted through the optical path and split one for each arm of the BS. $\tau_{i}$ is the overall optical transmittance of the photons in the $i$ th channel, and $2 \xi_{B S}\left(1-\xi_{B S}\right)$ accounts for the probability of splitting one photon on each BS arm. Finally $\left[1-\left(1-\eta_{2}\right)^{k}\right]$ is the probability that the trigger detector clicks in the presence of $k$ photons, with $\eta_{2}$ as its detection efficiency. By performing the calculation in Eq. (A2), we obtained

$$
p_{2}=1-\mathcal{P}\left(0 \mid 2 \xi_{B S}\left(1-\xi_{B S}\right) \tau_{1} \tau_{2} \eta_{2} \Lambda_{c} T\right) .
$$

In the low-triggering photon regime, i.e., $2 \xi_{B S}(1$ $\left.-\xi_{B S}\right) \tau_{1} \tau_{2} \eta_{2} \Lambda_{c} T \ll 1$, we can approximate Eq. (A3) with the first term of the series expansion, either $\mathcal{P}\left(n \mid \Lambda_{c} T\right)$ is a Poissonian or a thermal distribution, as

$$
p_{2} \simeq 2 \xi_{B S}\left(1-\xi_{B S}\right) \tau_{1} \tau_{2} \eta_{2} \Lambda_{c} T .
$$

The second step is to calculate the total coincidence probability $p_{c}^{t o t}$ per gate pulse. We assume that true coincidences due to correlated photons occur after the first half of the coincidence window $w$. Here we define $\pi_{w, \mathrm{I}}$ as the probability for the triggered detector not to fire for events arriving in the first half of $w$.

The triggered detector can fire given a trigger detector click. The trigger detector click may be due, as before stated, to the arrival of one photon of the pair $\left(p_{2}\right)$ or to accidental counts $\left(p_{2}^{a c c}\right)$.

We sort out the contributions to the total coincidence probability, considering which kind of event caused the trigger detector to fire, as following:

$$
\begin{aligned}
p_{c}^{t o t}= & p_{2}\left[\left(1-\pi_{w, \mathrm{I}}\right)+\pi_{w, \mathrm{I}} \eta_{1}+\pi_{w, \mathrm{I}}\left(1-\eta_{1}\right)\left(1-\pi_{w, \mathrm{II}}\right)\right] \\
& +\left(p_{2}^{a c c}+p_{2}^{d c}\right)\left(1-\pi_{w, \mathrm{I}} \pi_{w, \mathrm{II}}\right) .
\end{aligned}
$$

Therefore the first group in Eq. (A5) corresponds to the case of the trigger detector firing for a photon of the pairs, while the second group corresponds to the case of the trigger detector firing for accidental counts.

Within the first group in Eq. (A5) we distinguished the first term as the probability that, even if the trigger clicks for a photon of the pair, the triggered detector clicks for accidental events in the first half of the coincidence window $w$; the second term as the probability that the triggered detector does not fire in the first half of the coincidence window and clicks for the photon correlated $\left(\eta_{1}\right.$, the detection efficiency of the triggered detector $\left.\mathrm{APD}_{1}\right)$; the third term as the probability that the triggered detector does not fire in the first half of the coincidence window, does not fire for the correlated photon, but fires for an accidental event occurring in the second half of the coincidence window, where $\pi_{w, \mathrm{I}}\left(\pi_{w, \mathrm{II}}\right)$ is the probability for the triggered detector not to fire for events arriving in the first (second) half of $w$.

The second group is made of the probability of the triggered detector firing for accidental events, with the trigger firing for dark counts or photons which can produce only accidental coincidences.

It is straightforward to isolate the probability of true coincidences, obtaining

$$
p_{c}^{\text {true }}=p_{2} \pi_{w, \mathrm{I}} \eta_{1} .
$$

Then, by substituting expression (A4) in Eq. (A6) and solving for $\Lambda_{c}$ we obtain Eq. (1).

\section{ACKNOWLEDGMENTS}

This work has been partially supported within the following plants. National Council Research (CNR) and Ministry of Instruction, University, and Research (MIUR) plan (16/10/2000 FISR Funds CU 03.00204). 


\section{REFERENCES}

1. D. N. Klyshko, Photons and Nonlinear Optics (Gordon and Breach, 1988).

2. A. L. Migdall, S. Castelletto, I. P. Degiovanni, and M. L. Rastello, "Intercomparison of a correlated-photon-based method to measure detector quantum efficiency," Appl. Opt. 41, 2914-2922 (2002).

3. W. Tittel, J. Brendel, H. Zbinden, and N. Gisin, "Quantum cryptography using entangled photons in energy-time Bell states," Phys. Rev. Lett. 84, 4737-4740 (2000).

4. E. Knill, R. Laflamme, and G. J. Milburn, "A scheme for efficient quantum computation with linear optics," Nature 409, 46-52 (2001)

5. A. Migdall, "Correlated-photon metrology without absolute standards," Phys. Today 52, 41-46 (1999).

6. S. V. Polyakov and A. L. Migdall, "High accuracy verification of a correlated-photon-based method for determining photoncounting detection efficiency," Opt. Express 15, 1390-1407 (2007).

7. M. Pelton, P. Marsden, M. T. D. Ljunggren, and A. Karlsson, "Bright, single-spatial-mode source of frequency non-degenerate, polarization-entangled photon pairs using periodically poled KTP,” Opt. Express 12, 3573-3580 (2004)

8. M. A. Albota and E. Dauler, "Single photon detection of degenerate photon pairs at $1.55 \mu \mathrm{m}$ from a periodically poled lithium niobate downconverter," J. Mod. Opt. 51, 1417-1432 (2004).

9. C. E. Kuklevicz, M. Fiorentino, G. Messin, F. N. Wong, and J. H. Shapiro, "High-flux source of polarization-entangled photons from a periodically poled $\mathrm{KTiOPO}_{4}$ parametric downconverter," Phys. Rev. A 69, 013807 (2004).

10. J. Soderholm, K. Hirano, S. Mori, S. Inoue, and S. Kurimura, "Analysis of the generation of photon pairs in periodically poled lithium niobate," in Proceedings of the 8th International Symposium on Foundations of Quantum Mechanics in the Light of New Technology (World Scientific, 2006), pp. 46-49.

11. C. Kurtsiefer, M. Oberparlieter, and H. Weinfurter, "Highefficiency entangled photon pair collection in type-II parametric fluorescence," Phys. Rev. A 64, 023802 (2001).

12. F. A. Bovino, P. Varisco, A. M. Colla, G. Castagnoli, G. Di Giuseppe, and A. V. Sergienko, "Effective fiber-coupling of entangled photons for quantum communication," Opt. Commun. 227, 343-348 (2003).

13. S. Castelletto, I. P. Degiovanni, V. Schettini, and A. Migdall, "Spatial and spectral mode selection of heralded single photons from pulsed parametric down-conversion," Opt. Express 13, 6709-6722 (2005).

14. T. B. Pittmann, B. C. Jacobs, and J. D. Franson, "Heralding single photons from pulsed parametric down-conversion," Opt. Commun. 246, 545-550 (2005).

15. K. Sanaka, K. Kawahara, and T. Kunga, "New highefficiency source of photon pairs for engineering quantum entanglement," Phys. Rev. Lett. 86, 5620-5623 (2001).

16. K. Banaszek, A. B. U'Ren, and I. A. Walmsley, "Generation of correlated photons in controlled spatial modes by downconversion in nonlinear waveguides," Opt. Lett. 26, 1367-1369 (2001).

17. A. U'Ren, C. Silberhorn, K. Banaszek, and I. Walmsley, "Efficient conditional preparation of high-fidelity single photon states for fiber-optic quantum networks," Phys. Rev. Lett. 93, 093601 (2004).

18. S. Tanzilli, H. D. Riedmatten, W. Tittel, H. Zbinden, P. Baldi, M. D. Micheli, D. Ostrowsky, and N. Gisin, "Highly efficient photon-pair source using periodically poled lithium niobate waveguide," Electron. Lett. 37, 26-28 (2001).

19. A. Trifonov, A. Zavriyev, V. Denchev, and A. Leverrier, "Improving the performance of quantum key distribution apparatus," J. Mod. Opt. 54, 9-13 (2006).

20. S. Mori, J. Soderholm, N. Namekata, and S. Inoue, "On the distribution of $1550-\mathrm{nm}$ photon pairs efficiently generated using a periodically poled lithium niobate waveguide," Opt. Commun. 264, 156-162 (2006).

21. O. Alibart, S. Tanzilli, D. Ostrowsky, and P. Baldi, "Highperformance guided-wave asynchronous heralded singlephoton source," Opt. Lett. 30, 1539-1541 (2005).

22. I. Avrutsky and A. V. Sergienko, "Design of integrated optical source of twin photons," Phys. Rev. A 71, 033812 (2005).

23. J. Chen, K. F. Lee, C. Liang, and P. Kumar, "Fiber-based telecom-band degenerate-frequency source of entangled photon pairs," Opt. Lett. 31, 2798-2800 (2006).

24. P. Kok, W. J. Munro, K. Nemoto, T. C. Ralph, J. P. Dowling, and G. J. Milburn, "Linear optical quantum computing," arXiv:quant-ph/0512071 (2006).

25. P. Baldi, M. Sundheimer, K. E. Hadi, M. P. de Micheli, and D. B. Ostrowsky, "Comparison between differencefrequency generation and parametric fluorescence in quasiphase-matched lithium niobate stripe waveguides," IEEE J. Sel. Top. Quantum Electron. 2, 385-395 (1996).

26. C. Becker, T. Oesselke, J. Pandavenes, R. Ricken, K. Rochhausen, G. Schreiber, and W. Sohler, "Advanced Ti:Er: $\mathrm{LiNbO}_{3}$ waveguide lasers," IEEE J. Sel. Top. Quantum Electron. 6, 101-113 (2000).

27. T. Del Rosso, G. Margheri, S. Sottini, S. Trigari, M. De Sario, F. Prudenzano, and D. Grando, "An optical thermometer exploiting periodically poled lithium niobate for monitoring the pantographs of high-speed trains," IEEE Sens. J. 7, 417-425 (2007).

28. X. F. Cao, R. V. Ramaswamy, and R. Srivastava, "Characterization of annealed proton exchanged $\mathrm{LiNbO}_{3}$ waveguide for nonlinear frequency conversion," J. Lightwave Technol. 10, 1302-1313 (1992).

29. D. Grando, F. Gelli, S. Trigari, and S. Sottini, "Caratterizzazione di guide a scambio protonico su Niobato di Litio polato periodicamente o per mezzo di impulsi elettrici o per Titanio indiffuso" in Proceedings of the Convegno Nazionale sulle Tecniche Fotoniche nelle Telecomunicazioni (Atti di Fotonica 2001), pp. 207-201.

30. S. Castelletto, I. P. Degiovanni, V. Schettini, and A. Migdall, "Optimizing single-photon-source heralding efficiency and detection efficiency metrology at $1550 \mathrm{~nm}$ using periodically poled lithium niobate," Metrologia 43, S56-S60 (2006).

31. id 200 Single-Photon Detector Module, Application Note, id Quantique, Switzerland (2004).

32. G. Hocker and W. K. Burns, "Mode dispersion in diffused channel waveguides by effective index method," Appl. Opt. 16, 113-118 (1977).

33. Guide to the Expression of Uncertainty in Measurement (International Organization for Standardization, 1995).

34. E. Kapon and R. Bath, "Low-loss single-mode GaAs/AlGaAs optical waveguides grown by organometallic vapor phase epitaxy,” Appl. Phys. Lett. 50, 1628-1630 (1987).

35. T. Del Rosso, "Dispositivi in $\mathrm{LiNbO}_{3}$ periodicamente polarizzato per applicazioni alla sensoristica ed alle telecomunicazioni," Ph.D. thesis (U. Florence, 2006). 